\title{
Effect of Q-factor manipulation via pedal spacers on lower limb frontal plane kinematics during cycling
}

\author{
Andrew N. Fife', Harsh H. Buddhadev', David N. Suprak', Sarah B. Paxson², Jun G. San \\ Juan $^{1}$ \\ 1 Department of Health and Human Development, Western Washington University, Bellingham, \\ Washington, United States of America \\ 2 CorePhysio, Bellingham, WA
}

* Correspondence: Harsh H. Buddhadev. harsh.buddhadev@wwu.edu

Received: 1 October 2019; Accepted: 11 March 2019; Published: 30 June 2020

\begin{abstract}
Anecdotal evidence suggests that frontal plane kinematics of the lower extremity are an important aspect of bicycle fit, however, frontal plane adjustments are often overlooked during common fitting procedures. The purpose of this study was to manipulate $\mathrm{Q}$-factor width via pedal spacers to determine their influence on frontal plane kinematics of the hip, knee, and ankle during cycling. Twenty-four young healthy recreational cyclists (12 female) completed five minutes of pedaling at their preferred cadence and power output under three stance widths conditions: no spacer, $20 \mathrm{~mm}$ spacer, and $30 \mathrm{~mm}$ spacer. For each participant, the pedaling cadence and power output were kept identical for all experimental conditions. Lower extremity marker position data were captured at $250 \mathrm{~Hz}$ for the last two minutes of each condition. Sixty consecutive crank cycles were analyzed to identify maximum and minimum hip, knee, and ankle angles in the frontal plane. With an increase in Q-factor, hip and knee maximum abduction angles increased and maximum adduction angles decreased. With increase in Q-factor from no spacer to $20 \mathrm{~mm}$ spacer condition, hip abduction increased by $0.8^{\circ}(\Delta 10 \% ; \mathrm{p}<0.001)$ whereas hip abduction decreased by $0.9^{\circ}(\Delta 23 \% ; \mathrm{p}<0.001)$ and similarly, knee abduction increased by $1.2^{\circ}$ $(\Delta 60 \% ; \mathrm{p}=0.002)$ whereas knee abduction decreased by $1.1^{\circ}(\Delta 18 \% ; \mathrm{p}=0.003)$. And with increase in Q-factor from no spacer to $30 \mathrm{~mm}$ spacer condition, hip abduction increased by $1.4^{\circ}(\Delta 18 \%$; $\mathrm{p}<0.001)$ and hip adduction decreased by $1.6^{\circ}(\Delta 40 \%$; $\mathrm{p}<0.001)$ and similarly, knee abduction increased by $1.8^{\circ}(\Delta 86 \% ; \mathrm{p}<0.001)$ and knee adduction decreased by $2.1^{\circ}(\Delta 35 \% ; \mathrm{p}<0.001)$. Maximum and minimum ankle angles were not affected by the stance width conditions $(\mathrm{p}>0.05)$. Pedal spacers are an effective way of manipulating Q-factor and frontal plane kinematics of the hip and knee and could help cyclists experiencing medial or lateral knee pain.
\end{abstract}

Keywords: knee adduction; bicycle fit; Q-factor; pedaling

\section{Introduction}

Cycling is frequently used during rehabilitation in a variety of clinical settings as well as for fitness and recreation (Mangione et al. 1999; Ridgel et al. 2012; Salacinski et al. 2012). It is critical that the bike-fit is accurately performed to minimize joint pain and improve comfort
(Silberman et al. 2005). Lower extremity motion during cycling primarily occurs in the sagittal plane. Hence, joint motion in this plane has been studied extensively (Elmer et al. 2011; Ericson et al. 1986; Marsh et al. 2000) and when

performing a bike fit, focus has traditionally involved manipulations in the sagittal plane (Bini et al. 2014; Bini et al. 2010; Silberman et al.

(c) 2020 Fife, licensee JSC. This is an Open Access article distributed under the terms of the Creative Commons Attribution License ((http://creativecommons.org/licenses/by/4.0) which permits unrestricted use, distribution, and reproduction in any medium, provided the original work is properly cited. 
2005). During the bike fitting procedure, lower extremity position in the frontal plane is often overlooked and under-researched. Silberman et al. (2005) explains the importance of frontal plane foot position during cycling. They state that if the feet are too close together a cyclist could experience lateral knee pain. Whereas if their feet are too far apart, they can experience medial knee pain. To our knowledge, there is very limited research on the effect of manipulating foot position in the frontal plane on lower extremity joint motion during cycling (Disley and Li 2014b; Thorsen et al. 2019).

An effective and convenient method to manipulate stance width on a cycle ergometer is the use of pedal spacers (McCutcheon et al. 2008). Pedal spacers are machined components that insert between the crankset and the pedal spindle. Interfacing pedal spacers between the pedal and crank effectively increases mediolateral distance between pedals and thus may influence frontal plane knee joint motion and postural alignment (i.e., reduce knee adduction angles) (Thorsen et al. 2019). Pedal spacers can be purchased in multiple lengths (e.g., $20 \mathrm{~mm}, 30$ $\mathrm{mm}$ ) to manipulate stance width on bicycles to different extents. Currently, there are several pedal spacer options available commercially with claims that these pedal spacers may reduce knee adduction position during cycling; however, there is limited research supporting these claims (Thorsen et al. 2019).

In a recent study, Thorsen et al. (2019) examined the effect of Q-factor manipulation on frontal plane knee joint kinematics and kinetics at different fixed work rates at a controlled cadence (i.e., $80 \mathrm{~W}, 120 \mathrm{~W}$, and $160 \mathrm{~W}$ at $80 \mathrm{rpm}$ ). They found that peak knee abduction angles and peak knee abduction moments increased with increases in Q-factor. With changes in frontal plane position of the feet induced by different Qfactors, it is possible that hip and ankle frontal plane motion could also be affected, however, this has not been examined. Researchers sometimes experimentally control work rate and cadence (as done by Thorsen et al. (2019)) to ensure the high internal validity of the experiment (i.e., all participants pedaled at identical work rates and cadences). However, these conditions may not reflect the natural performance of the participants. In the current study, preferred work rate and cadence were chosen to enhance the external validity of the experiment. Thus, the novel aspects of the current study are the examination of changes in frontal plane hip, knee, and ankle angles with Qfactor manipulation at a self-selected work rate and cadence.

Therefore, the purpose of this study was to examine the effect of $Q$-factor manipulation, via different lengths of pedal spacers, on lower extremity frontal plane joint motion in healthy young adults. We hypothesized that maximum hip, knee, and ankle joint angles would systematically become more abducted as QFactor increased.

\section{Methods}

\section{Participants}

Twelve female (Mean (SD): 24.5 (3.7) years; 167.4 (5.0) $\mathrm{cm} ; 60.8(6.9) \mathrm{kg})$ and 12 male (22.3 (3.5) years; $183.8(7.0) \mathrm{cm} ; 84.2(15.7) \mathrm{kg}$ ) participants were recruited from the campus of Western Washington University and the local community. G*Power 3.1 software was used calculate sample size based on the large effect size of Q-factor on maximum knee adduction angle reported by Thorsen et al. (2019). A minimum sample size of 8 participants was needed to achieve a statistical power of 0.8 to detect a large effect size main effect $(\eta 2=0.601)$ of pedal spacers on lower extremity joint angles at an alpha level of 0.05 . Recreational cyclists were classified as people who self-reported that they had ridden a bicycle for recreation, transportation, and/or exercise throughout the majority of the past year (Buddhadev and Martin 2018). Participants with pain or recent injuries to the lower extremity were excluded. Almost all participants self-identified as being recreational mountain bikers. The University Institutional Review Board approved the study design and procedures and all participants provided an informed consent prior to participation. The study meets the ethical standards outlined by the International Journal of Sports Medicine (Harriss and Atkinson 2011). 


\section{Data Collection}

Each participant completed one testing session that lasted approximately 75 minutes. Prior to the arrival of the participants, the capture volume for a six-camera Vicon system (Vicon Vero, Centennial, CO, USA) was calibrated and the calibration error always remained under $0.5 \mathrm{~mm}$. The six Vicon Vero cameras had a resolution of 2.2 megapixels and were used to collect data at $250 \mathrm{~Hz}$. All cycling tests were carried out on an electronically braked Velotron Dynafit ergometer (Racer-Mate Inc, Seattle, WA, USA), which allowed for seat and handlebar position adjustment as well as the ability to control power output as cadence varied. The cycle ergometer was also calibrated prior to all data collection sessions by executing the Accuwatt calibration check test (Racer-Mate, Inc, Seattle, WA, USA).(Buddhadev et al. 2018) The ergometer calibration deviated no more than $0.5 \%$ from the factory settings across all data collection sessions. Participants completed the informed consent and a health history and cycling experience questionnaire upon arrival to determine if they met the inclusion and exclusion criteria for the study. Each participant was provided with lycra cycling shorts, a tank top, and appropriately sized cycling shoes (Giro, Santa Cruz, CA, USA) that clipped into the pedals (Shimano, Sakai, Japan). Anthropometric characteristics such as body height, body mass, inter-anterior superior iliac spine (ASIS) distance, and bilateral leg length, knee width, and ankle width were measured. Digital calipers (Vinca, Clockwise tools, Santa Clarita, CA, USA) were used to measure knee and ankle widths. Twenty-four retroreflective $14 \mathrm{~mm}$ markers were placed on both lower extremities using a modified lower extremity Plug-in Gait model from Vicon. The modification to the model included adding 2 markers each to the pelvis, thigh, shank, and foot segments. These modification were needed to recreate markers that were likely to be covered as the participants adopted a cycling posture and to identify individual crank cycles. To ensure consistency, the same researcher (ANF), with a year of experience with Plug-in gait marker placement, performed all the marker placement. Using the
Vicon motion capture system, marker position data were captured at a sampling frequency of 250 Hz. Static trials were collected with participants standing in motorcycle pose (i.e., standing with arms abducted to $90^{\circ}$ and forearms flexed to $90^{\circ}$, such that no markers were covered by the arms during the static trial) according to the Vicon Plug-in Gait guidelines (VICON 2017). A cycling ergometer fit procedure was then implemented to standardize the cycling posture of the participants and control the effects of posture on the dependent variables (Bailey et al. 2003; Fang et al. 2016; Shen et al. 2018). A handheld goniometer (Bikefit, Kirkland, WA, USA) was used to measure joint angles during the bike fitting procedure. Seat height was set with the crank positioned at bottom dead center and the knee flexed to $30^{\circ}$ (Ferrer-Roca et al. 2012; Priego Quesada et al. 2017; Silberman et al. 2005). Seat fore-aft position was adjusted such that when the crank arms were horizontal, a plumb line dropped from the inferior pole of the patella of the forward leg rested directly over the pedal spindle (Buddhadev et al. 2018; Buddhadev and Martin 2018; Silberman et al. 2005). Finally, handlebar position was adjusted and confirmed using a goniometer to achieve a forward trunk angle of $30^{\circ}$ from the vertical plane to make cycling more comfortable (Buddhadev et al. 2018; Buddhadev and Martin 2018).

Participants then performed a 5-minute warm-up and familiarization on the ergometer at their selfselected power and cadence. This familiarization also served to determine the preferred power output and cadence of the participants, which was used for the three experimental conditions. Specifically, during the first 2 minutes of the warm-up the participants pedaled at their natural cadence and asked the researchers to increase the work rate until they found a work rate they preferred to comfortably pedal at their selfselected cadence. After they had identified their preferred work rate and cadence, they were asked to cycle at that work rate and cadence for the remaining 3 minutes of the warm-up and familiarization to confirm and ensure that those were their preferred work rates and cadences. At the end of the 5-minute warm-up, the researcher recorded the participant's preferred work rate 
and cadence data. For the experimental conditions, the data was collected using the "manual ergo mode" on the electronically braked Velotron ergometer. In this mode, the researcher enters the preferred work rate value of the participant in Velotron CS software interfaced with the ergometer. This software fixes the work rate of the ergometer to that value. During the experimental conditions, the participants pedaled precisely at their preferred work rate and kept their cadence at their previously identified selfselected cadence value (using concurrent feedback about their cadence from the computer screen positioned directly in front of the ergometer). The $\mathrm{Q}$ factor (i.e., the horizontal distance between outer side of left and right crank (Thorsen et al. 2019) of the no-spacer condition was $150 \mathrm{~mm}$ wide as per manufacturer specifications, which was confirmed with digital calipers (Vinca, Clockwise tools, Santa Clarita, CA, USA). Generally, the Q-factor on a road bike is approximately $150 \mathrm{~mm}$ and on a mountain bike is $180 \mathrm{~mm}$ (Disley and Li 2014a).

Preferred work rate and cadence were chosen to enhance the external validity of the experiment. When researchers experimentally control work rate (i.e., keep it fixed e.g. $75 \mathrm{~W}$ ), they are often criticized that the fixed work rate may not reflect the natural work rate of the participant and thus, the data collected may not reflect the natural performance of the participants. Furthermore, the most important independent variable in the experiment was the manipulation of Q-factor via pedal spacers. The analysis of the effect of $Q-$ factor manipulation on frontal plane hip, knee, and ankle joint angles would be a within-subjects comparison. Thus, by choosing preferred workrates we examined the effects Q-factor manipulation on lower extremity frontal plane joint angles at the participants natural preferred cycling performance (i.e., their preferred work rates and cadences). Please note, we had male and female participants in the experiment simply to make the data generalizable to both sexes.

Each participant performed three experimental stance width conditions (no pedal spacers, $20 \mathrm{~mm}$ pedal spacers, and $30 \mathrm{~mm}$ pedal spacers (Kneesavers $^{\mathrm{TM}}$, North Hills, California, USA)), with the order of completion randomly assigned.
Participants completed each condition by pedaling at their preferred power output and cadence (determined in the familiarization session) for five minutes. During this 5-minute cycling, after the second minute the participants were asked to rate their comfort of cycling using an enlarged 0 to $10 \mathrm{~mm}$ Visual Analog Scale, where a larger number indicated greater discomfort (Gardner et al. 2015; Thorsen et al. 2019). Marker position data during the experimental conditions were captured during the last two minutes of the five minutes of each condition. A 3-5-minute rest interval separated the conditions during which the pedals were prepared for the subsequent condition. After each fitting, the pedals were tightened to a torque of 45 $\mathrm{Nm}$ with a calibrated torque wrench based on the manufacturer guidelines.

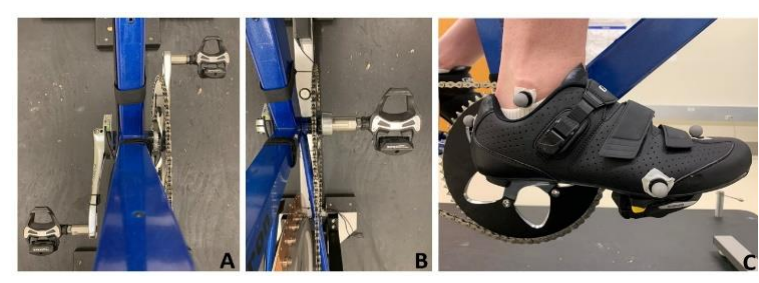

Figure 1. Ergometer setup. Figure 1A: Largest Qfactor $(210 \mathrm{~mm})$ configuration; Figure 1B: Pedal with the $30 \mathrm{~mm}$ spacer, and; Figure 1C: Marker placement on the foot.

\section{Data analysis}

Marker position data were filtered at $4 \mathrm{~Hz}$ using a 4th order Butterworth filter (Elmer et al. 2011; Gardner et al. 2016; Shen et al. 2018). For the no spacer experimental condition, we first cropped the trial to include 4 cycles of data. Then we manually labelled all the markers for those time frames. Then for gaps 10 frames or less (i.e., $<0.04$ seconds), we did a spline fill. For 11-30 frames gap, we used pattern fill for the thigh, shank, and foot data. For the pelvis markers, we used rigid body fill to fill gaps. Fortunately, we did not have gaps larger than 30 frames. After the gap filling, we completed the step of auto-initialize labelling and did a dynamic calibration of the trial with 4cycles of data. After the dynamic calibration, we applied the calibration to full trials of all three experimental conditions and we had very minimal gaps. Again, for gaps 10 frames or less 
we did a spline fill. And for 11-30 frames gap, we used pattern fill for the thigh, shank, and foot data. For the pelvis markers, we used rigid body fill to fill gaps. We did not have gaps larger than 30 frames.

The dynamic Plug-in Gait pipeline was executed to determine the frontal plane hip, knee, and ankle joint angles (VICON 2017). The joint angles were calculated in flexion/extension, abduction/adduction, and internal/external rotation sequence. In the frontal plane for the right leg, adduction angles were positive and abduction angles were negative. Top dead centers (TDC) of individual crank cycles were identified using markers positioned on the feet in close proximity to the left and right pedal spindles. A reflective marker was placed on 5th metatarsal of the foot (which was positioned directly above the pedal spindle; see figure 1C). During a crank cycle, the highest vertical position of this marker coincided with the top-dead center of an individual crank cycle. Using the computed TDC positions, joint angle data for 60 consecutive crank cycles were identified to attain stable estimates of kinematic data. The joint angle data were imported in MATLAB. For each crank cycle, maximum and minimum angles were identified and ranges (i.e., the difference between the maximum and minimum angle for each joint) were determined for hip, knee, and ankle frontal plane angles and averaged across 60 cycles for the statistical analysis.

\section{Statistical analysis}

First, one-way analysis of variance (ANOVA) was performed to determine the effects of sex on the dependent variables. If there were no differences in the dependent variables based on sex, then one-way ANOVAs with repeated measures were used to evaluate the effects of stance width manipulation via pedal spacers (3) on the frontal plane hip, knee, and ankle maximum and minimum angles and ROM, and cycling comfort. If the dependent variables were influenced by sex, then a two-way (sex $x$ condition) mixed model ANOVA was performed to determine the effects of sex and condition on the dependent variables. Alpha level was set a priori at 0.05. A Greenhouse-Geisser correction was performed if the assumption of sphericity was violated. For significant main effects of condition and sex-condition interaction, post-hoc analyses were performed with t-tests. For main effects and interaction analysis, paired-tests were used for within-subjects contrasts and independent samples $t$-test were used for between-subjects contrasts. Effect size was calculated as partial eta squared ( $\eta \mathrm{p} 2)$. Partial eta squared was interpreted using guidelines provided by Vincent where, $\eta p 2>0.01$ was small, $\eta p 2>0.06$ was medium, and $\eta p 2>0.15$ was large (Vincent 1999). Statistical analyses were conducted using SPSS (version 21; IBM Corporation, Armonk, NY).

\section{Results}

\section{Knee frontal plane motion}

For knee frontal plane motion, the knee angle became more abducted from TDC to BDC and then returned to a more adducted position when the crank cycle was completed (figure $2 b$ ). There were no sex-condition interactions for the knee frontal plane maximum angles or $\operatorname{ROM}(\mathrm{p}>0.05$; Table 1). There was a significant main effect of condition for maximum knee frontal plane angles $(F 2,44=20.786 ; p<0.001 ; \eta p 2=0.486)$. The knee angle became systematically less adducted as pedal stance width increased from no spacer condition to $20 \mathrm{~mm}(\mathrm{p}=0.003)$ and 30 $\mathrm{mm}(\mathrm{p}<0.001)$ spacer conditions. The magnitude of decrease in the maximum knee adduction angles from no spacer to $20 \mathrm{~mm}$ spacer condition was $0.7-1.4^{\circ}(\mathrm{p}=0.003)$, when examining male and female data together. There was also a reduction in maximum knee adduction angle from $20 \mathrm{~mm}$ to $30 \mathrm{~mm}$ spacer conditions $\left(0.7-1.4^{\circ} ; \mathrm{p}=0.012\right)$. Conversely, knee abduction angles increased with increase in pedaling stance width $(\mathrm{F} 2,44=19.261 ; \mathrm{p}<0.001$; $\eta p 2=0.467)$. Compared to the no spacer condition, the magnitude of maximum knee abduction angles were $1.2^{\circ}$ greater for the 20 mm spacer $(p=0.002)$ and $1.8^{\circ}$ greater for the 30 $\mathrm{mm}$ spacer conditions $(\mathrm{p}<0.001)$. With increase in stance width from $20 \mathrm{~mm}$ to $30 \mathrm{~mm}$ spacer condition, the magnitude of maximum knee 
abduction increased $0.5^{\circ}$, but this difference was not statistically significant $(p=0.078)$.

There was also a significant main effect of sex on both maximum and minimum knee adduction angles. For male participants, the maximum adduction angle was $8.5^{\circ}$ more than females $(\mathrm{F} 1,22=10.548 ; \mathrm{p}=0.004 ; \eta p 2=0.324)$. Conversely, the female maximum knee abduction angle was $5.8^{\circ}$ more than males ( $\mathrm{F} 1,22$ $=6.241 ; \mathrm{p}=0.020 ; \eta p 2=0.224$ ).

\section{Hip frontal plane motion}

For the hip frontal plane motion, the hip angle remained in an abducted position throughout the crank cycle (figure 2c). The hip was in an abducted position at TDC and moved towards an adducted position during the first $60^{\circ}$ of the crank cycle. The hip then moved toward a more abducted position at BDC before returning to a less abducted position at TDC.

For hip frontal plane ROM, there was a sexcondition interaction (F2,44=3.269; $\mathrm{p}=0.047$; $\eta \mathrm{p} 2=0.129$ ). Post-hoc comparisons showed that there were no differences in male and female participants' hip frontal ROM for any of the conditions. In addition, for male participants there was no change in hip frontal ROM across the conditions. However, for female participants there was a $0.5^{\circ}$ difference in hip ROM between no spacer and $20 \mathrm{~mm}$ spacer condition for female participants $(\mathrm{p}=$ 0.040). For maximum and minimum hip adduction angles, there was no sexcondition interaction or a sex main effect ( $p>0.05$ ). There was a significant main effect of condition. Hip frontal plane minimum (F2,44 $=46.068 ; \mathrm{p}<0.001 ; \eta \mathrm{p} 2$ $=0.677)$ and maximum (F2,44 = 36.271; $\mathrm{p}<0.001 ; \eta \mathrm{p} 2=0.622)$ angles became systematically more abducted as pedal stance width increased from the no spacer condition to $20 \mathrm{~mm}$ and $30 \mathrm{~mm}$ spacer conditions. The magnitude of this shift in frontal plane maximum angles ranged from $1.4-1.6^{\circ}$.

\section{Ankle frontal plane motion}

The ankle angle remained in a slightly inverted position throughout the crank cycle (figure 2a). A condition main effect was also observed for ankle frontal plane $\mathrm{ROM}(\mathrm{F} 2,44=4.254 ; \mathrm{p}=$ $0.020 ; \eta p 2=0.162$ ). Compared to the no spacer condition, the $30 \mathrm{~mm}$ spacer condition was $0.062^{\circ}$ more everted $(p=0.020)$. Also, $30 \mathrm{~mm}$ spacer was $0.062^{\circ}$ more everted $(p=0.011)$ than the $20 \mathrm{~mm}$ spacer conditions. These differences of $0.06^{\circ}$ are perhaps beyond the precision of the motion capture system's measurement capacity and thus, we think these differences are not meaningful. There was no effect of sex or pedal spacer conditions on the frontal plane ankle maximum and minimum angles. For the ankle frontal plane ROM, a sex main effect was observed (F1,22 = 7.191; $\mathrm{p}=0.014 ; \eta \mathrm{p} 2=0.246)$. The ankle position of the female participants were slightly more adducted $\left(\sim 0.6^{\circ}\right)$ compared than male participants.
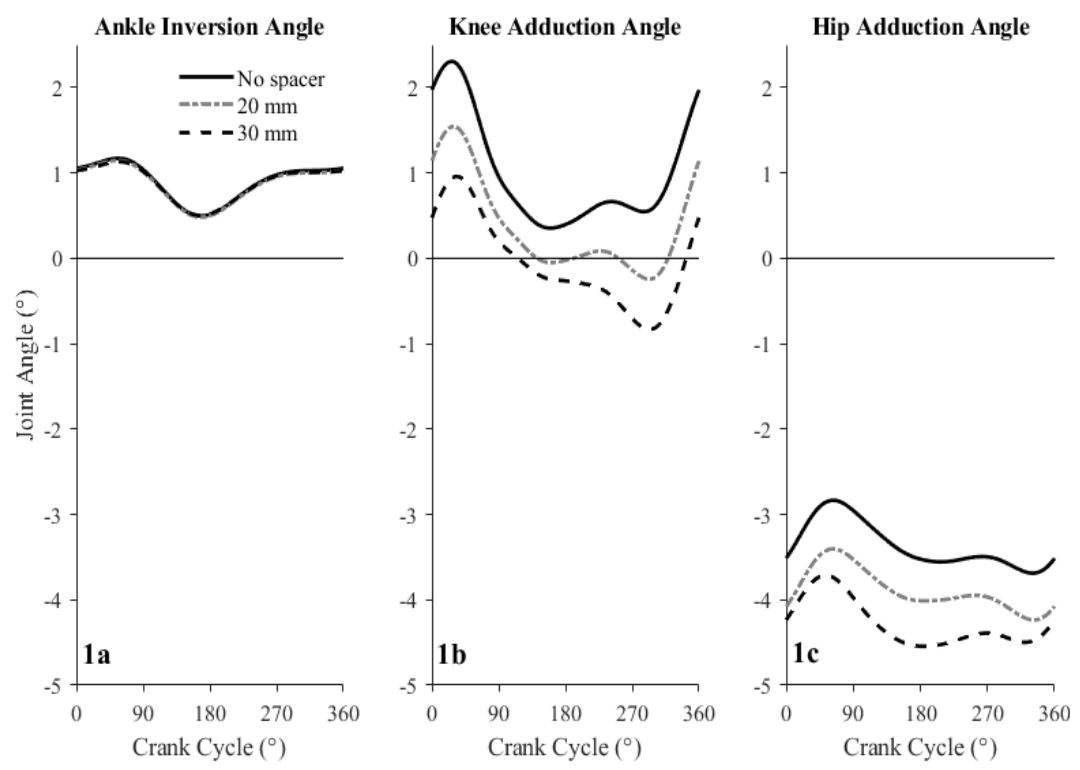

Figure 2. Frontal plane joint angles ensembled over 60 consecutive crank cycles for all participants (male and female). Positive angles represent ankle inversion and knee and hip adduction. 
Table 1. Effect of stance width condition on lower extremity frontal plane joint kinematics

\begin{tabular}{|c|c|c|c|c|c|c|}
\hline & & Female & & & Male & \\
\hline & $\begin{array}{l}\text { No } \\
\text { spacer }\end{array}$ & $\begin{array}{l}20 \mathrm{~mm} \\
\text { spacer }\end{array}$ & $\begin{array}{l}30 \mathrm{~mm} \\
\text { spacer }\end{array}$ & $\begin{array}{l}\text { No } \\
\text { spacer }\end{array}$ & $\begin{array}{l}20 \mathrm{~mm} \\
\text { spacer }\end{array}$ & $\begin{array}{l}30 \mathrm{~mm} \\
\text { spacer }\end{array}$ \\
\hline Maximum Hip Adduction $\left({ }^{\circ}\right)$ * & $-7.8(2.8)$ & $-8.7(2.3)$ & $-9.5(2.5)$ & $-8.1(4.1)$ & $-8.9(4.3)$ & $-9.3(4.2)$ \\
\hline Minimum Hip Adduction $\left({ }^{\circ}\right)$ * & $-3.4(2.9)$ & $-4.7(2.7)$ & $-5.2(3.0)$ & $-4.5(4.2)$ & $-5.1(3.9)$ & $-5.9(3.9)$ \\
\hline Hip Frontal $\operatorname{ROM}\left({ }^{\circ}\right)^{\wedge}$ & $4.5(1.3)$ & $4.0(1.2)$ & $4.3(1.6)$ & $3.6(1.3)$ & $3.8(1.5)$ & $3.4(1.4)$ \\
\hline $\begin{array}{l}\text { Maximum Knee Adduction }\left(^{\circ}\right) \# \text {, } \\
\text { * }\end{array}$ & $1.7(5.6)$ & $0.3(6.1)$ & $-0.4(5.9)$ & $9.9(6.7)$ & $9.2(7.8)$ & $7.9(6.3)$ \\
\hline $\begin{array}{l}\text { Minimum Knee Adduction }\left(^{\circ}\right) \# \text {, } \\
\text { * }\end{array}$ & $-4.7(6.1)$ & $-6.3(6.8)$ & $-6.8(7.0)$ & $0.6(4.2)$ & $-0.3(5.2)$ & $-0.8(4.1)$ \\
\hline Knee Frontal ROM $\left(^{\circ}\right)$ & $6.3(2.4)$ & $6.5(2.4)$ & $6.5(2.4)$ & $9.4(4.9)$ & $9.5(4.9)$ & $8.6(4.8)$ \\
\hline Maximum Ankle Inversion $\left(^{\circ}\right)$ & $2.6(1.8)$ & $2.5(1.9)$ & $2.5(1.9)$ & $1.8(1.5)$ & $1.7(1.6)$ & $1.8(1.5)$ \\
\hline Minimum Ankle Inversion $\left(^{\circ}\right)$ & $1.0(1.9)$ & $0.9(1.9)$ & $0.9(2.0)$ & $0.7(1.7)$ & $0.7(1.8)$ & $0.8(1.7)$ \\
\hline Ankle Frontal $\operatorname{ROM}\left(^{\circ}\right) \#, *$ & $1.6(0.6)$ & $1.6(0.5)$ & $1.6(0.6)$ & $1.1(0.5)$ & $1.1(0.5)$ & $1.0(0.4)$ \\
\hline
\end{tabular}

Note. Angles are Mean (SD); negative angles are abduction and positive angles are adduction; * statistically significant stance width condition main effect; \# statistically significant sex main effect; $\wedge$ statistically significant sex-condition interaction.

Note, there was no difference in pelvis width (i.e., horizontal inter-ASIS) between female and male participants (Female: 25.1 (2.6) cm; Male: $26.3(1.2) \mathrm{cm}$ ). The preferred power outputs and cadences for female participants were 91.1 (31.4) Watts (W) and 73.5 (12.3) revolutions per minute (rpm), respectively. For male participants, the preferred outputs and cadences were 120.7 (34.5) $\mathrm{W}$ and 69.8 (7.8) rpm, respectively.
With respect to comfort during cycling, there were no statistically significant sex-condition interaction $(\mathrm{F} 2,44=0.589 ; \mathrm{p}=0.559 ; \eta \mathrm{p} 2=0.026)$ or main effects for either sex $(\mathrm{F} 1,22=0.078$; $\mathrm{p}=$ $0.783 ; \eta p 2=0.004)$ or spacer conditions $(\mathrm{F} 2,44=$

1.154; $\mathrm{p}=0.325 ; \eta p 2=0.050)$. For female participants, cycling comfort for no spacer condition was $0.75 \pm 1.77,20 \mathrm{~mm}$ spacer condition it was $0.42 \pm 1.17$, and for $30 \mathrm{~mm}$ condition it was $0.75 \pm 0.87$. Similarly for male participants, the cycling comfort for no spacer, $20 \mathrm{~mm}$ spacer, and $30 \mathrm{~mm}$ spacer conditions were $0.58 \pm 0.90,0.67 \pm 0.78,0.10 \pm 1.21$, respectively. 


\section{Discussion}

The purpose of this study was to examine the effect of pedal stance manipulation, via different lengths of pedal spacers, on lower extremity frontal plane joint motion in healthy young adults. We hypothesized that maximum hip, knee, and ankle joint angles would systematically become more abducted as QFactor increased. The hip and ankle joint angle data support this hypothesis. Maximum hip and knee angles significantly became more abducted with increases in Q-Factor (no spacer $>20 \mathrm{~mm}$ spacer > $30 \mathrm{~mm}$ spacer; Table 1). These differences had large effect sizes but were small in magnitude (in degrees).

The data demonstrated that overall the lower extremity moved into a relatively more abducted position with increase in pedal stance width when compared to the no spacer condition (figure 2). The majority of the increases in the lower extremity abduction positions were observed at the hip and knee joints. When the data were examined together for both sexes, the magnitude of increase in the hip abduction angle was 1.4-1.6 ${ }^{\circ}$. Compared to the no spacer condition, these were $20 \%$ greater for the $20 \mathrm{~mm}$ spacer condition and $40 \%$ greater for the $30 \mathrm{~mm}$ spacer condition. Similarly, the knee adduction angle decreased with the stance width conditions by 1.1-2.1 ${ }^{\circ}$. Compared to the no spacer condition, the knee was $18 \%$ and $36 \%$ less adducted during the $20 \mathrm{~mm}$ and $30 \mathrm{~mm}$ spacer conditions, respectively. Overall, these changes appear to be very subtle in overall angle magnitude, but they account for a large percent change in maximum angles (Umberger and Martin 2001). The feet were clipped into the pedals which could have contributed to the little to no change in position or ROM of the feet at the ankle joint across the stance width conditions (Gregersen and Hull 2003; Umberger and Martin 2001). To the authors' knowledge, this is the first study to systematically examine lower extremity frontal plane joint positions and ROM for cycling at different stance widths.
The frontal plane joint ROM data showed that pedal spacer conditions affected the knee joint ROM most, followed by the hip and the ankle joint ROM. This hierarchy of effect on lower extremity joint ROM is understandable because the feet are fixed at the pedals and the proximal ends of the thighs are semi-fixed at the seat (Duc et al. 2008). The knees are the least constrained joints during the pedaling motion, which perhaps explains the relatively larger effect on the frontal plane ROM at this joint. Indeed, the largest increase in joint abduction position (in degrees) with increased stance width was also in the same order; the largest change in maximum angles occurred at the knee followed by the hip.

In the current study, as Q-factor was increased (QF 150-210 mm) the hip and knee frontal plane angles became systematically more abducted. These findings are consistent with recent research conducted by Thorsen et al. (2019), who reported increases in peak knee abduction angles and peak knee abduction moments with increases in Q-factor (QF 150-276 mm). They also reported that these increases in knee adduction angles and moments were accompanied by increases in lateral pedal reaction forces without a changes in vertical pedal reaction forces (Thorsen et al. 2019). These increases in lateral pedal reaction forces are intuitive because the feet move more laterally with increases in Qfactor. An interesting finding reported by Disley and Li (Disley and Li 2014a) was that systematic increase in Q-factor (QF 90-180 mm) did not affect magnitude and timing of muscular activation of Vastus Lateralis, Vastus Medialis, Tibialis Anterior, and Gastrocnemius Medialis muscles during cycling. The data from Thorsen et al. (2019) and Disley and Li (2014a) suggest that changes in frontal plane knee moments are a result of changes in pedal reaction forces and are not caused by changes in timing or activation of the knee extensor muscles.

The present study has several novel aspects that fill gaps and add to the research literature related to the effects of Q-factor on cycling performance. To our knowledge, the current study is the first to examine frontal plane 
kinematics of the hip and ankle, in addition to the knee, during stationary cycling at different Q-factors. Our data demonstrate that both knee and hip angles become more abducted as feet move apart with wider Q-factors. We did not observe any changes in ankle frontal plane angles because the feet were clipped-in to the pedals and thus, controlled motion at the ankle joint. In addition to these dependent variables, another novel aspect was that the study was conducted at self-selected work rate and cadence, which could be more reflective of participants' natural pedaling performance compared to pedaling at a fixed work rate and cadence. Regardless of self-selected or fixed pedaling conditions, data from both Thorsen et al.(2019) and our study show that maximum knee abduction angles increased with increases in Q-factor. Alternatively, the findings of systematic changes in frontal plane knee angles with increases in Q-factor are robust and perhaps independent of the selected work rate and cadence during cycling. Silberman et al.(2005) explains the importance of frontal plane foot position during cycling. They state that if the feet are too close together or too far apart, a cyclist may experience knee pain. Identifying acute adaptations in cycling patterns derived from wider stances can be used to address bike fit and perhaps injury reduction based on findings from the present study.

The frontal plane joint angle data for the no spacer condition observed in the current study are comparable to data reported previously (Gardner et al. 2016; Shen et al. 2018; Umberger and Martin 2001). In the current study, hip abduction maximum angles ranged from $4^{\circ}$ to $8^{\circ}$ which are comparable to $2^{\circ}$ to $8^{\circ}$ reported by Umberger and Martin (2001). Also, the frontal plane hip range of motion reported in the current study is comparable to hip frontal plane range of motion data reported by Bini et al. (2016). The knee frontal plane maximum angles in the current study ranged from $6^{\circ}$ of adduction to $2^{\circ}$ of abduction with a frontal plane ROM of $8^{\circ}$. Previous research reported similar maximum angles with ROM ranging between $10-12^{\circ}$ (Gardner et al. 2016; Shen et al. 2018; Thorsen et al. 2019; Umberger and Martin 2001). In the current study, the participants' feet were clipped into the pedals and therefore, ankle frontal ROM was under $2^{\circ}$, which was slightly lower than other studies (3-6 ${ }^{\circ}$ ) (Shen et al. 2018; Umberger and Martin 2001).

To allow for our data to be generalizable to both sexes, we recruited young male and female participants. The male participants were more relatively adducted at the knee compared to female participants throughout the crank cycle. The stance width conditions systematically affected frontal plane kinematics at the knee joint for both the sexes in a similar manner. Our data show that frontal plane knee joint angles differ based on sexes and therefore, when increasing stance width, the natural knee joint position of an individual should be considered. For example, adult males whose knees are in a relatively more adducted position during cycling could benefit from increasing stance width. Because the pedal spacers move the knee into a more abducted position, participants who have a more neutral or abducted knee position in the control condition, such as the female participants, should exercise caution when implementing wider pedal stance widths during cycling.

Changes in pedal stance width, via pedal spacers, affect frontal plane knee position. An important implication is that the changes in frontal plane knee position could indirectly influence the position and tracking of the patella in the patellar groove during cycling. If the pedal spacer condition caused malalignment of the patella (e.g., too abducted), it may increase the risk of knee ailments such as patellofemoral pain syndrome (Dettori and Norvell 2006; Herrington and Nester 2004). When manipulating frontal plane knee joint angle, a final position that places the knee in a more neutral posture may be favorable.

In conclusion, stance width condition systematically affected frontal plane knee joint position. Compared to the no spacer condition, the knee angle was less adducted for the $20 \mathrm{~mm}$ 
and $30 \mathrm{~mm}$ spacer conditions. In addition, the knee was less adducted for the $30 \mathrm{~mm}$ compared to the $20 \mathrm{~mm}$ spacer condition.

\section{Conflict of interest}

None.

\section{References}

1. Bailey MP, Maillardet FJ, Messenger N (2003) Kinematics of cycling in relation to anterior knee pain and patellar tendinitis. J Sports Sci 21: 649657

2. Bini RR, Dagnese F, Rocha E, Silveira MC, Carpes FP, Mota CB (2016) Three-dimensional kinematics of competitive and recreational cyclists across different workloads during cycling. Eur J Sport Sci 16: 553-559

3. Bini RR, Hume PA, Kilding AE (2014) Saddle height effects on pedal forces, joint mechanical work and kinematics of cyclists and triathletes. Eur J Sport Sci 14: 44-52

4. Bini RR, Tamborindeguy AC, Mota CB (2010) Effects of saddle height, pedaling cadence, and workload on joint kinetics and kinematics during cycling. Journal of Sports Rehabilitation 19: 301314

5. Buddhadev HH, Crisafulli DL, Suprak DN, San Juan JG (2018) Individuals with knee osteoarthritis demonstrate interlimb asymmetry in pedaling power during stationary cycling. J Appl Biomech 34: 306-311

6. Buddhadev HH, Martin PE (2018) Effects of age, power output, and cadence on energy expenditure and lower limb antagonist muscle coactivation during cycling. Journal of Aging and Physical Activity: 1-10

7. Dettori NJ, Norvell DC (2006) Non-traumatic bicycle injuries: A review of the literature. Sports Med 36: 7-18

8. Disley BX, Li FX (2014a) The effect of Q Factor on gross mechanical efficiency and muscular activation in cycling. Scand J Med Sci Sports 24: $117-121$

9. Disley BX, Li FX (2014b) Metabolic and kinematic effects of self-selected $Q$ factor during bike fit. Res Sports Med 22: 12-22
10. Duc S, Bertucci W, Pernin JN, Grappe F (2008) Muscular activity during uphill cycling: Effect of slope, posture, hand grip position and constrained bicycle lateral sways. J Electromyogr Kinesiol 18: 116-127

11. Elmer SJ, Barratt PR, Korff T, Martin JC (2011) Joint-specific power production during submaximal and maximal cycling. Med Sci Sports Exerc 43: 1940-1947

12. Ericson MO, Bratt A, Nisell R, Arborelius UP, Ekholm J (1986) Power output and work in different muscle groups during ergometer cycling. Eur J Appl Physiol 55: 229-235

13. Fang Y, Fitzhugh EC, Crouter SE, Gardner JK, Zhang S (2016) Effects of workloads and cadences on frontal plane knee biomechanics in cycling. Med Sci Sports Exerc 48: 260-266

14. Ferrer-Roca V, Roig A, Galilea P, García-Lopez J (2012) Influence of saddle height on lower limb kinematics in well-trained cyclists: static vs. dynamic evaluation in bike fitting. J Strength Cond Res 26: 3025-3029

15. Gardner JK, Klipple G, Stewart C, Asif I, Zhang S (2016) Acute effects of lateral shoe wedges on joint biomechanics of patients with medial compartment knee osteoarthritis during stationary cycling. J Biomech 49: 2817-2823

16. Gardner JK, Zhang S, Liu H, Klipple G, Stewart C, Milner CE, Asif IM (2015) Effects of toe-in angles on knee biomechanics in cycling of patients with medial knee osteoarthritis. Clin Biomech 30: 276-282

17. Gregersen CS, Hull ML (2003) Non-driving intersegmental knee moments in cycling computed using a model that includes threedimensional kinematics of the shank/foot and the effect of simplifying assumptions. J Biomech 36: $803-813$

18. Harriss DJ, Atkinson G (2011) Update--Ethical standards in sport and exercise science research. Int J Sports Med 32: 819-821

19. Herrington L, Nester C (2004) Q-angle undervalued? The relationship between $\mathrm{Q}$-angle and medio-lateral position of the patella. Clin Biomech 19: 1070-1073

20. Mangione KK, McCully K, Gloviak A, Lefebvre I, Hofmann M, Craik R (1999) The effects of highintensity and low-intensity cycle ergometry in 
older adults with knee osteoarthritis. J Gerontol A Biol Sci Med Sci 54: M184-190

21. Marsh AP, Martin PE, Sanderson DJ (2000) Is a joint moment-based cost function associated with preferred cycling cadence? J Biomech 33: 173-180

22. McCutcheon MJ, Jensen RL, Watts PB, Marlor RA (2008) Oxygen consumption: Effect of lateral pedal width variations relative to q-angle in avid cyclists. International Conference on Biomechancis in Sports, Seoul, South Korea

23. Priego Quesada JI, Pérez-Soriano P, LucasCuevas AG, Salvador Palmer R, Cibrián Ortiz de Anda RM (2017) Effect of bike-fit in the perception of comfort, fatigue and pain. J Sports Sci

24. Ridgel AL, Peacock CA, Fickes EJ, Kim CH (2012) Active-assisted cycling improves tremor and bradykinesia in Parkinson's disease. Arch Phys Med Rehabil 93: 2049-2054

25. Salacinski AJ, Krohn K, Lewis SF, Holland ML, Ireland K, Marchetti G (2012) The effects of group cycling on gait and pain-related disability in individuals with mild-to-moderate knee osteoarthritis: a randomized controlled trial. J Orthop Sports Phys Ther 42: 985-995

26. Shen G, Zhang S, Bennett HJ, Martin JC, Crouter SE, Fitzhugh EC (2018) Effects of knee alignments and toe clip on frontal plane knee biomechanics in cycling. Journal of Sports Science and Medicine 17: $312-321$

27. Silberman MR, Webner D, Collina S, Shiple BJ (2005) Road bicycle fit. Clin J Sport Med 15: 271276

28. Thorsen T, Strohacker K, Weinhandl JT, Zhang S (2019) Increased Q-Factor increases frontal-plane knee joint loading in stationary cycling. Journal of Sport and Health Science 2019: 1-7

29. Umberger BR, Martin PE (2001) Testing the Planar Assumption During Ergometer Cycling. 55-62

30. VICON (2017) Plug-in Gait Reference Guide

31. Vincent W (1999) Statistics in Kinesiology. Human Kinetics, Champaign, IL 